\title{
Laboratory analysis of amino acids, 2018 revision: a technical standard of the American College of Medical Genetics and Genomics (ACMG)
}

\author{
J. Daniel Sharer, $\mathrm{PhD}^{1}$, Irene De Biase, $\mathrm{MD}, \mathrm{PhD}^{2}$, Dietrich Matern, $\mathrm{MD}, \mathrm{PhD}^{3}$, Sarah Young, $\mathrm{PhD}^{4}$, \\ Michael J. Bennett, $\mathrm{PhD}^{5}$ and Adviye A. Tolun, $\mathrm{PhD}^{6}$; \\ on behalf of the ACMG Laboratory Quality Assurance Committee
}

\begin{abstract}
Disclaimer This laboratory standard is designed primarily as an educational resource for clinical laboratory geneticists to help them provide quality clinical laboratory genetic services. Adherence to this standard is voluntary and does not necessarily assure a successful medical outcome. This standard should not be considered inclusive of all proper procedures and tests or exclusive of other procedures and tests that are reasonably directed to obtaining the same results. In determining the propriety of any specific procedure or test, the clinical laboratory geneticist should apply his or her own professional judgment to the specific circumstances presented by the patient or specimen.

Clinical laboratory geneticists are encouraged to document in the patient's record the rationale for the use of a particular procedure or test, whether or not it is in conformance with this standard. They also are advised to take notice of the date any particular standard was adopted, and to consider other relevant medical and scientific information that becomes available after that date. It also would be prudent to consider whether intellectual property interests may restrict the performance of certain tests and other procedures.
\end{abstract}

Amino acid abnormalities are observed in a broad spectrum of inherited metabolic diseases, such as disorders of amino acid metabolism and transport, organic acidemias, and ureagenesis defects. Comprehensive analysis of physiologic amino acids in blood, urine, and cerebrospinal fluid is typically performed in the following clinical settings: evaluation of symptomatic patients in whom a diagnosis is not known; evaluation of previously diagnosed patients to monitor treatment efficacy; evaluation of asymptomatic or presymptomatic (at-risk) relatives of known patients; follow-up testing for an abnormal newborn screen; and assessment of dietary protein adequacy or renal function in general patient populations. Currently, the most common analytical method to quantify amino acids is based on ion exchange chromatography using post-column derivatization with ninhydrin and spectrophotometric detection. Newer methodologies are based on liquid chromatographic separation with detection by mass spectrometry or spectrophotometry. Amino acid analysis by nonseparation methods, such as the flow injection-tandem mass spectrometric (MS/MS) method used for newborn screening, is considered inadequate for the diagnosis of at-risk patients. The purpose of this document is to provide a technical standard for amino acid analysis as applied to the diagnosis and management of inborn errors of metabolism.

Genetics in Medicine (2018) 20:1499-1507; https:/doi.org/10.1038/ s41436-018-0328-6

Keywords: clinical genetic testing; technical standards; amino acidopathies; amino acids

\section{Amino acids and proteins}

\section{BACKGROUND}

Amino acids serve as protein building blocks, metabolic intermediates, and substrates for energy production. By definition, amino acids contain an amino group and a carboxyl group, and often contain another functional group (e.g., sulfhydryl, hydroxyl, or secondary amino- or carboxylgroup). Proteins consist of 20 different amino acids, half of which are synthesized endogenously (nonessential), while the remaining amino acids are obtained from dietary sources (essential). For almost a century, the detection of amino acids depended on ninhydrin, a chemical that reacts specifically with primary and secondary amines to produce a purple color that can be measured spectrophotometrically. The development of the amino acid analyzer (based on ion exchange chromatographic separation of amino acids coupled with post-column ninhydrin derivatization) in the 1950s was an important advance that started large-scale investigations into

\footnotetext{
${ }^{1}$ Department of Genetics, University of Alabama at Birmingham School of Medicine, Birmingham, AL, USA; ${ }^{2}$ Department of Pathology, University of Utah School of Medicine, Salt Lake City, UT, USA; ${ }^{3}$ Department of Laboratory Medicine and Pathology, Mayo Clinic College of Medicine, Rochester, MN, USA; ${ }^{4}$ Department of Pediatrics, Duke School of Medicine, Durham, NC, USA; ${ }^{5}$ Department of Pathology and Laboratory Medicine, University of Pennsylvania Perelman School of Medicine and Children's Hospital of Philadelphia, Philadelphia, PA, USA; ${ }^{6}$ Laboratory Corporation of America ${ }^{\circledR}$ Holdings (LabCorp), Durham, NC, USA. Correspondence: J. Daniel Sharer (dsharer@uab.edu) 
inborn errors of metabolism. While only 20 amino acids are found in proteins, more than 100 amino acids occur in body fluids ${ }^{1}$ and at least 76 of these are of biological interest. ${ }^{2}$ Amino acids are essential for humans, and daily urinary losses are very small due to renal tubular reabsorption. Defects in amino acid metabolism and transport cause inborn errors of metabolism $^{3}$ and generalized aminoaciduria can be seen in patients with impaired kidney function. In addition, several amino acids act as neurotransmitters, and abnormal levels are significant for certain neurometabolic disorders. Because the normal concentrations of most amino acids in cerebrospinal fluid are quite low, analytical methods must be sensitive and accurate. As amino acid disorders include both catabolic and anabolic pathways, analysis should be capable of detecting pathophysiological elevations and reductions of amino acid levels. ${ }^{2,4}$

\section{General description of amino acidemias and acidurias}

Inherited defects of amino acid catabolism, biosynthesis, or transport have been known for many years, and new defects continue to be identified. ${ }^{5-9}$ Cystinuria (MIM 220100) and alkaptonuria (MIM 203500) were among the first inherited metabolic disorders described by Archibald Garrod. ${ }^{10}$ Phenylketonuria (PKU) (MIM 261600) (ref. ${ }^{11}$ ), the inherited amino acid disorder with the highest impact on public health, was first described in 1934 (ref. ${ }^{12}$ ) and prompted the initiation of routine newborn screening in the early 1960 s (ref. ${ }^{13}$ ).

Amino acid disorders are clinically and biochemically heterogeneous. They are characterized biochemically by the accumulation or dissipation of pathological amounts of normal metabolites, or metabolites that are not present under normal physiological conditions, but are produced from alternative pathways in response to loss of a specific gene product's function (typically an enzyme or transporter protein). Primary disorders of amino acid metabolism include defects in the catabolic pathways of aromatic amino acids (e.g., phenylketonuria and tyrosinemia), sulfur-containing amino acids (classic homocystinuria, MIM 236200), branched-chain amino acids (maple syrup urine disease, MIM 248600), urea cycle intermediates (including ornithine transcarbamylase deficiency, MIM 311250; classic citrullinemia, MIM 215700; and argininosuccinic aciduria, MIM 207900), and several others. The broad category of amino acid disorders also includes transport defects such as cystinuria (MIM 220100), lysinuric protein intolerance (LPI, MIM 22700), citrin deficiency (MIM 605814 and 603471), and Hartnup disorder (MIM 234500) (ref. ${ }^{3}$ ). Several other amino acid disorders are characterized by abnormally low amino acid concentrations caused by the inability to synthesize a nonessential amino acid. Examples include asparagine synthetase deficiency (MIM 615574) (ref. ${ }^{14}$ ) and glutamine synthetase deficiency (MIM 610015) (ref. ${ }^{15}$ ), as well as remethylation ${ }^{16}$ and serine deficiency disorders. ${ }^{17}$ Recently, a defect in branched-chain ketoacid dehydrogenase kinase $^{18}$ has been reported that is characterized by increased catabolism leading to abnormally low concentrations of branched-chain amino acids.

Clinical findings associated with amino acid disorders are diverse but are often disease-specific. Signs and symptoms include overwhelming illness resulting from systemic biochemical derangements such as hyperammonemia, hypoglycemia, metabolic acidosis, or respiratory alkalosis. Several amino acid disorders also cause neurologic abnormalities including seizures, hypotonia, lethargy, coma, developmental delay, unexplained intellectual disability, failure to thrive, or autistic behaviors. Depending on the particular disorder, patients may present from birth to adulthood with one or more organ system affected.

It is important to take into consideration that certain amino acid conditions (e.g., aminoacylase 1 deficiency [MIM 609924] and aromatic L-amino acid decarboxylase [AADC] deficiency [MIM 608643]) may not be amenable to detection by amino acid analysis due to the nature of the intermediates produced. In the case of aminoacylase 1 deficiency, abnormal urinary excretion of $\mathrm{N}$-acetylated L-amino acids can be detected by organic acid analysis using gas chromatography-mass spectrometry. Therefore, a comprehensive metabolic workup ideally should include at a minimum urine organic acid and plasma acylcarnitine analysis, in addition to amino acid analysis.

\section{Secondary amino acid abnormalities}

Amino acid abnormalities are not only associated with inborn errors of metabolism but are also sensitive markers of an individual's nutritional state and can indicate dysfunction of various organs including liver, kidney, and muscle. For example, liver is the primary organ for the metabolism of methionine and tyrosine, leading to their elevations in metabolic and nonmetabolic causes of hepatic dysfunction. Because acquired changes in amino acid concentrations may be subtle during the malfunctioning of a particular organ, amino acid analysis should have high accuracy and reproducibility to enable interpretation of these changes. ${ }^{2}$

\section{Incidence}

The combined incidence for all amino acidopathies is more frequent than 1 in 10,000 (ref. ${ }^{9}$ ). This estimate does not include other inborn errors of metabolism (e.g., organic acidemias or congenital lactic acidemias) that may need amino acid analysis for comprehensive diagnosis and treatment monitoring. The incidence of PKU in Caucasian populations is between 1 in 10,000 and 1 in 15,000 live births. ${ }^{19}$ The incidence of urea cycle disorders is estimated to be at least 1:35,000 births, ${ }^{20}$ with ornithine transcarbamylase deficiency being the most common urea cycle defect. ${ }^{21}$

\section{Mode of inheritance}

The majority of disorders of amino acid metabolism or transport are inherited as autosomal recessive traits. Exceptions include disorders such as X-linked ornithine transcarbamylase deficiency (MIM 311250), methionine adenosyltransferase 
deficiency (MIM 250850, an autosomal dominant form of hypermethioninemia caused by heterozygosity for the p. $264 \mathrm{H}$ variant in the MAT1A gene), ${ }^{22}$ and hawkinsinuria (MIM 140350, an autosomal dominant disorder of tyrosine metabolism caused by deficiency of 4-hydroxyphenylpyruvic acid dioxygenase). ${ }^{23}$

\section{METHODS}

The laboratory technical standard was informed by a review of the literature, including any current guidelines, and expert opinion. Resources consulted included PubMed (search terms: inborn errors of metabolism, amino acidopathies, amino acid analysis methods, ion exchange chromatography [\& amino acid], ultra-performance liquid chromatography [\& amino acid], tandem mass spectrometry [\& amino acid \& liquid OR gas chromatography]), the American College of Medical Genetics and Genomics (ACMG) Standards and Guidelines for Clinical Genetics Laboratories, Clinical and Laboratory Standards Institute (CLSI) guidelines, CLIA regulations, and the Centers for Disease Control and Prevention Morbidity and Mortality Weekly Report (MMWR) on Good Laboratory Practices for Biochemical Genetics Testing and Newborn Screening for Inherited Metabolic Disorders. When the literature provided conflicting evidence about a topic or when there was insufficient evidence, the authors used expert opinion to inform the recommendations. Expert opinion included the coauthors of the document, members of the ACMG Laboratory Quality Assurance Committee, as well as the experts consulted outside of the Committee but acknowledged in this document. Any conflicts of interest for workgroup members or consultants are listed. A draft was delivered to the ACMG Board of Directors for review and member comment. The draft document was posted on the ACMG website and an email link was sent inviting ACMG members to provide comment. All comments were assessed by the authors. When appropriate, additional evidence was included to address member comments and the draft was amended. Both member comments and author responses were reviewed by a representative of the ACMG Laboratory Quality Assurance Committee and by the ACMG Board of Directors. The final document was approved by the ACMG Board of Directors. This updated standard replaces the previous version in Section F: Clinical Biochemical Genetics, American College of Medical Genetics and Genomics Standards and Guidelines for Clinical Genetics Laboratories (2008 Edition, Revised 02/ 2007), section F7.5 (ref. ${ }^{24}$ ).

\section{PREANALYTICAL REQUIREMENTS Specimen requirements \\ Plasma is the preferred specimen for the evaluation of most primary disorders of amino acid metabolism. Lithium, or sodium, heparin is the preferred anticoagulant. Ethylenedia- minetetraacetate (EDTA) plasma yields similar results to heparinized plasma with minor differences that are typically}

not diagnostically significant. ${ }^{25-27}$ Yet, it should be noted that EDTA can react with ninhydrin and produce a ninhydrin positive contaminant that may lead to interference. ${ }^{27}$ Sodium citrate-treated blood yields lower values for most amino acids. Laboratories should have established procedures for procuring specimens using their preferred anticoagulant. Fasting plasma samples are recommended to avoid erroneous interpretation of several disorders, particularly those involving amino acid synthesis that are characterized by low concentrations of certain amino acids. ${ }^{2}$ Serum should not be used because clotting times may lead to analyte-specific artifacts. ${ }^{27}$ Testing can also be performed on amino acids eluted from dried-blood spots (DBS).

Urine is the appropriate specimen for the identification of compounds that are efficiently cleared by the kidney (e.g., argininosuccinic acid [ASA]) or specific disorders of renal amino acid transport (e.g., renal Fanconi syndrome or cystinuria). However, urine is generally less reliable for firsttier investigations of primary amino acid disorders due to effective tubular reabsorption of a majority of amino acids, and susceptibility to interference from medications. Urine amino acid analyses may often be ordered erroneously in place of, or unnecessarily in addition to, plasma amino acid testing; therefore clinical indications should be preemptively reviewed (if available). When urine is analyzed, it should be collected without preservatives or fecal contamination.

The analysis of cerebrospinal fluid (CSF) is useful for diagnosing several biochemical genetic disorders, including asparagine and glutamine synthetase deficiencies, serine deficiency disorders, and most notably, glycine encephalopathy (nonketotic hyperglycinemia [NKH; MIM 605899]). In $\mathrm{NKH}, \mathrm{CSF}$ should be collected and analyzed along with a simultaneously collected plasma sample, so that the CSF: plasma glycine ratio can be calculated..$^{28-30}$ It is noteworthy that CSF should be collected into preservative-free collection tubes; however, fluoride oxalate and lithium heparin tubes also suffice. CSF should be stored frozen if not immediately analyzed. Specimens contaminated with blood should be interpreted with caution as most amino acids are present in blood at much higher concentrations than in CSF. In such cases, a repeat CSF specimen may be advised.

\section{Sample handling, shipping, and storage}

Plasma should be centrifuged immediately to reduce the influence of other blood constituents on the free amino acid concentrations. Glutamate, aspartate, and taurine have high intracellular levels and increase by hemolysis. Plasma should be kept at $-20^{\circ} \mathrm{C}$ (for long-term storage, $-70{ }^{\circ} \mathrm{C}$ ) until analysis to slow the decomposition of glutamine. ${ }^{2}$ Serum, if used, should be centrifuged after the standard time allowed for clotting (usually 30 minutes). However, the added time for clotting can lead to artifacts from the deamination of arginine to ornithine by red blood cell arginase, and by the release of oligopeptides. Postprandial samples should be avoided and information about current medications (e.g., antibiotics or 
antiseizure medications) or dietary status may be informative. Following centrifugation, samples should be kept frozen $(-20$ ${ }^{\circ} \mathrm{C}$; for long-term storage, $-70^{\circ} \mathrm{C}$ ) until the time of analysis. CSF and urine specimens should be frozen as soon as possible after collection and stored frozen prior to analysis. Samples sent to the laboratory from an outside referral source should be shipped on dry ice via an overnight courier.

Finally, it is important to note that amino acids with terminal sulfhydryl groups (e.g., cysteine and homocysteine) readily form disulfide bonds leading to their association with plasma proteins, ${ }^{31,32}$ particularly albumin. ${ }^{33}$ Therefore, the accurate quantification of these amino acids necessitates separate techniques utilizing strong reducing agents. ${ }^{34,35}$ This is particularly true for plasma homocysteine, an important biomarker for disorders of the methionine cycle, folate and vitamin $\mathrm{B}_{12}$ metabolism, and a risk factor for cardiovascular disease.

\section{Preanalytical variables}

Sample collection and handling are discussed in the previous section. Interference from coeluting, ninhydrin-positive compounds occur with certain antibiotics (e.g., ampicillin), contrast dyes, and other medications. Amino acid concentrations can also be influenced by anticonvulsants (e.g., hyperglycinemia with valproate use), nutritional status (e.g., intravenous [IV] nutrition or starvation), clinical status (e.g., fever, infections, and liver or kidney dysfunction), treatment of acute lymphoblastic leukemia (ALL) using L-asparaginase, ${ }^{36,37}$ as well as bacterial contamination. These factors may affect results and should be taken into account when interpreting results.

\section{Clinical indications for testing}

Clinical presentations of different disorders of amino acid metabolism are variable and often nonspecific. Onset of symptoms may occur in the neonatal period or as late as adulthood. Amino acid analysis should be considered in a wide variety of clinical situations, such as:

1. Lethargy, coma, seizures, or vomiting in a neonate

2. Hyperammonemia

3. Failure to thrive

4. Electrolyte abnormalities, including metabolic acidosis or respiratory alkalosis

5. Lactic acidemia

6. Unexplained developmental delay or intellectual disability

7. Abnormal newborn screening results

8. A previously diagnosed sibling

9. Clinical presentation suggestive of a specific amino acid disorder

10. Monitoring treatment efficacy (e.g., dietary) of known patients

Amino acid analysis can also be used for dietary monitoring (including assessment of dietary protein adequacy or renal function) of metabolic patients on protein restriction and/or special formulas, irrespective of pathway affected, or in the general patient population not affected with an inherited metabolic disorder. Indeed, specific amino acid analytes can be used as biomarkers to assess the risk of developing more common medical conditions, including diabetes ${ }^{38}$ and cardiovascular disease, ${ }^{39}$ although these applications are outside the scope of these Standards.

The laboratory should be aware of the clinical indication(s) for testing, including the need for immediate analysis in a critically ill patient, so that testing can be triaged, results interpreted appropriately, and additional testing recommended as needed. Depending on the clinical situation, amino acid analysis is often performed together with urine organic acids, plasma carnitine (free and total), and plasma acylcarnitine profile analysis as part of a comprehensive metabolic evaluation. Ideally, these tests should all be performed by the same laboratory, and results integrated into a complete interpretation.

\section{METHOD VALIDATION}

\section{Calibration and quantitation}

The quantitation of amino acid concentrations should be performed in relation to known reference or calibration standards. Amino acid calibration mixtures are available from several commercial sources. Some manufacturers have omitted certain relatively unstable amino acids (e.g., asparagine) from these mixtures; in these cases, freshly prepared solutions of the missing compounds can be added to the commercial standards to form a complete set. Performance of calibration material should be verified either with weighed standards, or with amino acid calibrators obtained from an independent source (e.g., National Institute of Standards and Technology [NIST]). Instrument calibration should be verified, and/or the instrument should be recalibrated, at regular intervals as established by the laboratory per manufacturer's recommendations, and as required by CLIA ' 88 . This should also occur with the introduction of new columns, reagent lots, or following service to the instrument or its components.

Quantitation using ion exchange chromatography (IEC) or ultraperformance or high-performance liquid chromatography (U/HPLC) coupled with spectrophotometric ${ }^{40}$ or fluorometric $^{41}$ detection should be performed using at least one internal standard (IS). The selected standard should elute at a unique position in the chromatogram and not interfere with the analysis of other nearby compounds. Quantitative results should be calculated using an IS method that adjusts for the amount of IS in the patient sample relative to that in the calibration mixture. ${ }^{25}$ For methods that employ mass spectrometric detection, stable isotope analogs for each amino acid should be used as ISs when available. The lack of ISs for specific analytes may reduce the performance of stable isotope dilution, mass-spectrometric methods for those analytes. ${ }^{42}$ For the majority of amino acids with clinical significance, stable isotope standards are commercially available. The laboratory should establish protocols to determine and periodically verify their method's linear range, analytical 
measurement range, and lower limit of detection (see CLSI document C24-A3: Clinical and Laboratory Standards Institute. Statistical Quality Control for Quantitative Measurement Procedures: Principles and Definitions; Approved Guideline-Third Edition). Procedures should be in place for analytical values that fall outside of an assay's performance limits.

\section{Reference ranges}

Laboratory-specific reference ranges should be determined for each sample type (plasma, DBS, urine, and CSF), using guidelines as defined by a laboratory's policy and procedures for test validation (e.g., CLSI document EP28-A3c: Defining, Establishing, and Verifying Reference Intervals in the Clinical Laboratory; Approved Guideline-Third Edition). It is important to note that amino acid levels obtained from different sample types (e.g., plasma versus DBS) are not comparable and should not be used interchangeably for monitoring purposes (e.g., phenylalanine). Anonymized samples from a general patient population are acceptable for generating a laboratoryspecific reference range when patients with an identified diagnosis are excluded. These ranges should be categorized by age and sex when appropriate and verified on a regular interval as required by CLIA ' 88 . When literature-based ranges are used, they should be verified using laboratory-specific methods. Because amino acid levels vary significantly with age, reference intervals should be age-specific.

\section{Testing personnel}

Laboratory personnel performing quantitative amino acid analysis should be documented to have received appropriate training and demonstrated competency in the performance of the test. In addition, laboratory personnel should satisfy CLIA ' 88 requirements for high-complexity testing and have, at a minimum, an associate degree in a laboratory science or medical laboratory technology from an accredited institution. More comprehensive requirements may apply to laboratories in some US states (see General Policies B: Personnel Policies of the ACMG Standards and Guidelines for Clinical Genetics Laboratories, 2008 Edition, Revised 02/2007 (https://www. acmg.net/acmg/Publications/Standards___uidelines/

Personnel_Policies.aspx).

\section{ANALYSIS OF AMINO ACIDS \\ Analytical methods Overview of methods}

To analyze free amino acids in physiological specimens, deproteinization of the sample is necessary. For comprehensive, quantitative amino acid analysis, this is commonly achieved by protein precipitation using sulfosalicylic acid (SSA) or trichloroacetic acid (TCA), followed by centrifugation, and/or filtration. Supernatants or filtrates are diluted with buffer of appropriate $\mathrm{pH}$ prior to analysis. For limited amino acid analysis such as for newborn screening by flow injection tandem mass spectrometry, extraction of amino acids from DBS is achieved using acetonitrile, methanol, or an admixture of these solvents. Sample preparation should include the addition of at least one IS to control for run-to-run variation in sample extraction and analysis. For urine, the creatinine concentration is measured and useful for adjusting the sample volume prior to analysis. Postanalysis, amino acid concentrations are also often normalized to creatinine molality (e.g., $\mathrm{mmol} / \mathrm{mol}$ creatinine). It is important to note that the Jaffé method, in which creatinine reacts with alkaline picrate to form a complex absorbing at $480-520 \mathrm{~nm}$, is still commonly used to determine creatinine, despite well-recognized interference by bilirubin, protein, ketones, ketoacids, fatty acids, and other compounds. ${ }^{43}$ HPLC $^{44}$ and particularly liquid chromatography-tandem mass spectrometry (LC-MS/MS) ${ }^{45}$ methods for measuring creatinine offer significant improvements in sensitivity and specificity over the Jaffé method. Urine amino acid levels can also be reported from a 24-hour specimen collection (e.g., $\mu \mathrm{mol} / 24$ hours).

Analysis of the full physiologic amino acid profile can be achieved using several methods. ${ }^{46}$ IEC with post-column derivatization using ninhydrin and spectrophotometric detection has been the most widely performed method for several decades and is still a commonly used clinical laboratory method. Alternative methods have been developed that take advantage of the selectivity of mass spectrometry and the reduced analytical time of ultraperformance liquid chromatography. When coupled with techniques such as ultraperformance liquid chromatography or gas chromatography, higher throughput and enhanced performance of amino acid analysis has been reported. ${ }^{40,47-50}$ Such methods also require less sample volume when compared with IEC. However, it is recognized that mass spectrometric methods with additional complexities present relatively more challenges to laboratories that do not have the necessary expertise. Furthermore, each methodology may present a different source of variance (e.g., recovery of amino acids may be affected by the solvent used to precipitate protein in plasma samples). Thus, it is the laboratories' responsibility to determine the possible pitfalls of their specific test and to validate their methodology to address possible challenges and limitations.

A number of different instrument configurations are commercially available for amino acid analysis; these instruments have published methodologies that should be validated in the individual laboratory. Also see General Policies C7: Levels of Development of a Diagnostic Test, and C8: Test Validation of the ACMG Standards and Guidelines for Clinical Genetics Laboratories, 2008 Edition, revised 02/2007 (https:// www.acmg.net/acmg/Publications/Standards__Guidelines/ General_Policies.aspx).

\section{Ion exchange chromatography (IEC)}

IEC is the most commonly used method of amino acid analysis and recent method updates have improved observed matrix effects. ${ }^{51}$ IEC requires derivatization of amino acids for detection. This can be accomplished prior to separation of amino acids (pre-column derivatization) using ophthalaldehyde (OPA) or phenylisothiocyanate (PITC), or 
following separation (post-column derivatization) using ninhydrin.

Post-column ninhydrin derivatization has been the preferred method for many years by a majority of laboratories, although methods based on newer technologies are now gaining in popularity. In this method, compounds are simultaneously detected at wavelengths of $570 \mathrm{~nm}$ and 440 $\mathrm{nm}$ using a dual-wavelength spectrophotometer. Laboratories may report values from the $570 \mathrm{~nm}$ channel only, from the sum of the two channels, or from a combination of both (e.g., hydroxyproline and proline from the $440 \mathrm{~nm}$ channel, and the remainder of the amino acids from the $570 \mathrm{~nm}$ channel). Identification of amino acids following chromatographic separation is based on retention time. Most systems are capable of resolving and quantifying roughly 40 amino acid peaks in a typical patient sample (the exact number varies as some systems do not resolve all amino acids from neighboring peaks). Laboratories, however, may elect to report a smaller subset of clinically relevant compounds.

Each chromatogram should be visually inspected for run performance, as well as for atypical peaks that may not be included in peak identification tables, including $\Delta$-1-pyrroline-5-carboxylate, homocitrulline, ASA, and alloisoleucine (especially when the ratio of branched-chain amino acids is disturbed). For ninhydrin-based systems with spectrophotometric detection, an identification based on retention time comparison can be supported by standard spiking and by calculating the ratio of response at $570 \mathrm{~nm}$ to that at $440 \mathrm{~nm}$. The 570/440 ratio can be established for all standard compounds, and compared with patient values to confirm peaks where coeluting interfering substances are suspected. In determining these ratios, peak baselines should be carefully examined such that potential artifacts from baseline fluctuations are eliminated. ${ }^{52}$ Alternatively, where coelution is known to occur for amino acids that are diagnostically significant (e.g., homocitrulline and methionine on certain IEC systems), reinjection of the sample and analysis using an alternative gradient method may be useful to verify identity. Additionally, ASA identification can be confirmed by heating the sample at $90^{\circ} \mathrm{C}$ and observing the change of retention time with anhydrous ASA formation. Quantitation should be based on the recovery of the IS in each specimen compared with the recovery of the IS in the calibration mixture.

\section{Ultra Performance Liquid Chromatography (UPLC)}

UPLC is increasingly being utilized due to its rapid separation of amino acids in approximately 35 minutes compared with 2 hours for a typical IEC analysis. One example of an UPLC application for plasma, serum, CSF, and urine amino acids analysis is a method that utilizes pre-column derivatization with 6-aminoquinolyl-N-hydroxysuccinimidyl carbamate. This reagent rapidly reacts with both primary and secondary amines to produce stable chromophores that can be detected by ultraviolet spectrophotometry at $260 \mathrm{~nm}$. Derivatized samples have a stable baseline, superb peak separation, and excellent peak symmetry for all amino acids including those that elute at the end of analysis. Using norvaline as an IS, correlation of this assay with IEC was satisfactory with adequate detection of most significant amino acids. ${ }^{40}$ The superior resolution of UPLC compared with IEC confers better sensitivity for this method. Currently, the only known negative characteristic of this technique is the inability to separate ASA from ethanolamine with published methods. Laboratories using UPLC should consider validating a secondary analysis for specifically measuring ASA to confirm a suspected elevation. Noteworthy is the observation that alloisoleucine is separated from other branched-chain amino acids, negating the need for a special analysis to confirm the potential of maple syrup urine disease.

\section{Tandem mass spectrometry methods}

Within the last 15 years, methods utilizing liquid chromatography-electrospray-tandem mass spectrometry (LC-ESI-MS/MS) have been developed for amino acid analysis in plasma, urine, and CSF and applied to clinical practice. ${ }^{47,49,50,53-57}$ LC-ESI-MS/MS methods offer the advantages of increased specificity and throughput as compared with traditional IEC-UV/VIS methods. ${ }^{48}$ The main challenges for developing a robust LC-ESI-MS/MS amino acid test include the necessity to control for ion suppression or enhancement of the numerous analytes included in the analysis, and the separation of isobaric amino acid species. Additionally, these instruments usually require more frequent calibration than IEC-UV/VIS ${ }^{57}$ and data review and processing may be cumbersome. ${ }^{56}$

LC-ESI-MS/MS methods have been published for the analysis of underivatized or derivatized amino acids. These methods used UPLC or HPLC with one or more column chemistries, stable isotope dilution or isobaric tagging, and detection by selected reaction monitoring (SRM). ${ }^{46-50,54-56}$ Sensitivities as low as $1-2 \mu \mathrm{M}$ and linearity of up to $1000-2000 \mu \mathrm{M}$ have been reported. ${ }^{47,49,50,55-57}$ Unlike IEC, LC-ESI-MS/MS methods are subject to matrix effects, and thus require the use of stable isotope, internal standards (IS) to compensate for variable recoveries and ionization efficiencies. ${ }^{42,58}$ A stable isotope IS for each analyte is preferred; however, depending on the method, this may not be feasible. The lack of an IS may affect accuracy and precision for a specific analyte and is an important consideration for each laboratory. Published methods that employ stable isotope dilution often utilize commercially available $\left[{ }^{2} \mathrm{H}_{2}\right]$ to $\left[{ }^{2} \mathrm{H}_{8}\right]$ labeled amino acids for IS. ${ }^{47,49,54}$ It is generally accepted that IS with a considerable degree of deuterium label may not reliably reduce all matrix effects in every sample due to the slight alterations in physicochemical properties, leading to small shifts in retention time and differences in ionization. ${ }^{58,59}$ For several published LC-ESIMS/MS methods, stable isotope-labeled analogs were not utilized for every amino acid measured, necessitating the application of surrogate IS for a subset of analytes. For these reasons, while LC-ESI- MS/MS methods have compared well with established amino acid analytical methods, there are limitations with the measurement of some target analytes. 
The sample preparation procedure for underivatized, LCMS/MS methods ${ }^{47,49,50,57,60}$ is similar to IEC post-column derivatization methods in most cases: samples are combined with ISs and plasma proteins are precipitated using sulfosalicylic acid or organic solvent. Separation of underivatized amino acids has been achieved using reverse-phase chromatography with ion pairing reagents, ${ }^{47,49,50,60}$ and with hydrophilic interaction chromatography using an amide UPLC column ${ }^{57}$ for the analysis of 26 to 52 amino acids with 18 - to 30-minute run times. As noted above, full quantification using stable isotope dilution was performed for only a subset of clinically significant amino acids, with reduced performance of several analytes without a corresponding IS. Other challenges with these underivatized methods included inferior performance of highly polar amino acids that are poorly retained on a reverse-phase column, ${ }^{49}$ the requirement for extensive reconditioning of ion-pairing reverse-phase systems, ${ }^{47}$ and the lack of separation of Dalloisoleucine from isoleucine with methods utilizing hydrophilic chromatography. ${ }^{57}$

A published LC-ESI-MS/MS method that utilizes aminereactive isobaric tagging reagents $\left(\mathrm{iTRAQ}^{\circledR}{ }^{\circledR}\right.$; later aTRAQ ${ }^{\mathrm{TM}}$ Reagent 121) is an alternative approach to using stable isotope IS. $^{55,56}$ These reagents were initially used for multiplexed quantification of peptides and have been applied to measuring physiological amino acid concentrations. ${ }^{53}$ These methods are based on the differential derivatization of IS with an isotopically unlabeled reagent (2,5-dioxopyrrolidin-1-yl-2(4methylpiperazin-1-yl)acetate) and the derivatization of sample with ${ }^{13} \mathrm{C}_{6},{ }^{15} \mathrm{~N}_{2}$ - stable isotope labeled reagent to generate isobaric tags. The differentially labeled sample and IS have identical chromatographic retention, and can be separated by MS/MS by the generation of product ions with an $\mathrm{m} / \mathrm{z}$ difference of $8 \mathrm{amu}$. Pre-derivatized standards are combined with the sample after derivatization and serve to control for differences in injection volume and ionization efficiency. Two nonphysiologic amino acids are added to the sample before derivatization for monitoring sample extraction and derivatization efficiency. Analyte quantification can be obtained by comparison with IS as provided in specified amounts in each commercial kit. Alternatively, quantification using calibrators extracted with each run, and normalized using the ISs, improves accuracy by reducing variability due to different IS lots or tagging efficacy. ${ }^{55,56}$ Chromatography is performed using a C18 $(5 \mu \mathrm{m}, 4.6 \mathrm{~mm} \times 150 \mathrm{~mm})$ column with a total analysis time of 18 minutes $^{55,56}$ with efficient separation of isobaric amino acids with the exception of alloisoleucine and isoleucine; for the accurate quantification of these branchedchain amino acids, it is necessary to modify the method to include the detection of the underivatized alloisoleucine and isoleucine, and the use of an underivatized stable isotope-labeled IS. ${ }^{46,55,56}$ Thus, an aliquot is removed from the sample prior to the labeling (derivatization) reaction, mixed with IS, and recombined with the derivatized extract prior to injection on the column. An advantage of isobaric tagging compared with isotope dilution is the availability of a commercial kit that contains IS for each of the 42 analytes. $^{55,56}$

The possible pitfalls of a specific LC-ESI-MS/MS method of choice should be determined by the laboratory and the assay should undergo a thorough and systematic validation before use in a clinical laboratory.

\section{Quality control}

Quality control (QC) material should be evaluated at two different concentrations for all reported amino acids, and should be derived with the same matrix as patient specimens. QC material should be analyzed along with each patient batch. In situations where samples are run continuously (e.g., adding samples as they arrive to an ongoing queue, rather than in discrete batches), QC samples should be run daily (every 24 hours) per College of American Pathologists (CAP, Inspector Checklist CBG 12800). Thresholds for acceptance or rejection of a QC sample result, and remedial actions in the event of a QC failure, should be established and documented by the laboratory. QC data should be regularly monitored for trends that may affect test performance, and problems should be documented and remediated as appropriate. Thresholds for appropriate IS response should be established, and IS responses for each specimen should be monitored as another level of quality control. It is recommended to consider basic statistical QC analyses for clinical testing to enhance the performance of laboratory methods ${ }^{61}$ (see also CLSI document EP28-A3c: Defining, Establishing, and Verifying Reference Intervals in the Clinical Laboratory; Approved Guideline-Third Edition).

\section{Proficiency testing}

Participation in biannual proficiency testing (PT) activities is an important element of any laboratory quality assurance program. A PT program that evaluates both analytical and interpretive/diagnostic proficiency of amino acid analysis is offered by CAP and supervised by the CAP/ACMG Genetic Biochemical and Molecular Genetic Resource Committee. Another excellent program is offered by the European Research Network for evaluation and improvement of screening, Diagnosis and treatment of Inherited disorders of Metabolism (ERNDIM; http://www.erndimqa.nl), in which analytical and interpretive (or clinical) proficiency is monitored through the regular distribution of PT.

\section{TEST INTERPRETATION AND REPORTING}

\section{Interpretation}

Clinical amino acid analyses should be interpreted by an American Board of Medical Genetics and Genomics (ABMGG)-certified clinical biochemical geneticist. Because normal amino acid concentrations vary with age, quantitative results should be compared with a properly defined reference age group. Interpretations of amino acid results are based upon relative amino acid levels, pattern recognition, and correlation of positive and negative findings, rather than on individual amino acids levels alone. Amino acid abnormalities 
or overall profiles should also be considered in the context of clinical findings and/or additional test results.

\section{Reporting}

Reports should contain appropriate patient and specimen information whenever available as described in the American College of Medical Genetics Standards and Guidelines for Clinical Genetics Laboratories, Sections 2.4, 2.41, and 2.42 (https://www.acmg.net/acmg/Publications/Standards_Guideli nes/General_Policies.aspx) and as specified by CLIA '88. Written reports should provide units of measure, agedependent, laboratory-specific reference ranges, and an interpretation of findings. When abnormal results are detected, the interpretation should include an overview of significant results, correlation to any available clinical information, elements of differential diagnosis, recommendations for additional testing or confirmatory studies (e.g., enzyme assay, molecular analysis), and a phone number to reach the reporting laboratory for any additional questions. Recommendations for follow-up evaluation, including referral to a metabolic specialist, should also be included when appropriate.

\section{ACKNOWLEDGEMENTS}

The authors gratefully acknowledge Russell Grant for critical reading of the manuscript. Members of the ACMG Biochemical Genetics Subcommittee and the Laboratory Quality Assurance Committee reviewed and endorsed the final document.

\section{DISCLOSURE}

The following authors direct laboratories that perform amino acid analysis as a fee-for service: J.D.S., I.D.B., D.M., S.Y., M.J.B., and A.A.T.

\section{REFERENCES}

1. Bremer HJ. Disturbances of amino acid metabolism: clinical chemistry and diagnosis. Urban \& Schwarzenberg: Munich, Germany, 1981.

2. Thöny B, Duran M, Gibson KM. Laboratory guide to the methods in biochemical genetics. Berlin: Springer, 2008.

3. Camargo SM, Bockenhauer D, Kleta R. Aminoacidurias: clinical and molecular aspects. Kidney Int. 2008;73:918-25.

4. Grier RE, Gahl WA, Cowan T, Bernardini I, McDowell GA, Rinaldo P. Revised sections F7.5 (quantitative amino acid analysis) and F7.6 (qualitative amino acid analysis): American College of Medical Genetics Standards and Guidelines for Clinical Genetics Laboratories, 2003. Genet Med. 2004;6:66-68

5. Scriver CR, Lamm P, Clow CL. Plasma amino acids: screening, quantitation, and interpretation. Am J Clin Nutr. 1971;24:876-90.

6. Scriver CR, Blau N, Duran M, Blaskovics ME, Gibson KM. Physician's guide to the laboratory diagnosis of metabolic diseases. Berlin: Springer, 2012.

7. Clarke JTR. A clinical guide to inherited metabolic diseases. Cambridge University Press: Cambridge, UK, 2005.

8. Zschocke J, Hoffmann GF. Vademecum metabolicum: diagnosis and treatment of inborn errors of metabolism. Milupa Metabolics: Friedrichsdorf, Germany, 2011.

9. Saudubray JM, van den Berghe G, Walter JH. Inborn metabolic diseases: diagnosis and treatment. Berlin: Springer, 2014.

10. Garrod AE. The Lancet. The incidence of alkaptonuria: a study in chemical individuality. Nutr Rev. 1975;33:81-83.

11. Williams RA, Mamotte CD, Burnett JR. Phenylketonuria: an inborn error of phenylalanine metabolism. Clin Biochem Rev. 2008;29:31-41.
12. Fölling $A$. Uber ausscheidung von phenylbrenztraubensaure in den harn als stoffwechselanomalie in verbindung mit imbezillitat. Hoppe-Seyler's Z Physiol Chem. 1934;277:169-76.

13. Guthrie R, Susi A. A simple phenylalanine method for detecting phenylketonuria in large populations of newborn infants. Pediatrics. 1963;32:338-43.

14. Ruzzo EK, Capo-Chichi JM, Ben-Zeev B, et al. Deficiency of asparagine synthetase causes congenital microcephaly and a progressive form of encephalopathy. Neuron. 2013;80:429-41

15. Haberle J, Gorg B, Rutsch F, et al. Congenital glutamine deficiency with glutamine synthetase mutations. N Engl J Med. 2005;353:1926-33.

16. lacobazzi V, Infantino V, Castegna A, Andria G. Hyperhomocysteinemia: related genetic diseases and congenital defects, abnormal DNA methylation and newborn screening issues. Mol Genet Metab. 2014;113: 27-33.

17. van der Crabben SN, Verhoeven-Duif NM, Brilstra EH, et al. An update on serine deficiency disorders. J Inherit Metab Dis. 2013:36:613-9.

18. Novarino G, El-Fishawy $P$, Kayserili $H$, et al. Mutations in BCKD-kinase lead to a potentially treatable form of autism with epilepsy. Science. 2012;338:394-7.

19. National Institutes of Health Consensus Development Panel. National Institutes of Health Consensus Development Conference Statement: phenylketonuria: screening and management, October 16-18, 2000. Pediatrics. 2001;108:972-82.

20. Ah Mew N, Lanpher BC, Gropman A, Chapman KA, Simpson KL, Summar ML. Urea cycle disorders overview. In: Pagon RA, Adam MP, Ardinger $\mathrm{HH}$, et al., eds. GeneReviews. University of Washington (Seattle) and the NCBI (Washington, DC) 1993-2016.

21. Lichter-Konecki $U$, Caldovic L, Morizono $H$, Simpson K Ornithine transcarbamylase deficiency. In: Pagon RA, Adam MP, Ardinger $\mathrm{HH}$, et al., eds. GeneReviews. University of Washington (Seattle) and the NCBI (Washington, DC) 1993-2016.

22. Marcao A, Couce ML, Nogueira C, et al. Newborn screening for homocystinuria revealed a high frequency of MAT I/III deficiency in Iberian Peninsula. JIMD Rep. 2015;20:113-20.

23. Tomoeda K, Awata H, Matsuura T, et al. Mutations in the 4hydroxyphenylpyruvic acid dioxygenase gene are responsible for tyrosinemia type III and hawkinsinuria. Mol Genet Metab. 2000;71: 506-10.

24. American College of Medical Genetics and Genomics. Standards and guidelines for clinical genetics laboratories. 2008. http://www.acmg.net PDFLibrary/Standards-Guidelines-Clinical-Biochemical-Genetics.pdf.

25. Hommes FA. Techniques in diagnostic human biochemical genetics: a laboratory manual. Wiley: New York, US, 1990.

26. Parvy PR, Bardet J, Kamoun PP. EDTA in vacutainer tubes can interfere with plasma amino acid analysis. Clin Chem. 1983;29:735.

27. Chuang CK, Lin SP, Lin YT, Huang FY. Effects of anticoagulants in amino acid analysis: comparisons of heparin, EDTA, and sodium citrate in vacutainer tubes for plasma preparation. Clin Chem. 1998;44: 1052-6.

28. Applegarth DA, Edelstein AD, Wong LT, Morrison BJ. Observed range of assay values for plasma and cerebrospinal fluid amino acid levels in infants and children aged 3 months to 10 years. Clin Biochem. 1979;12: 173-8.

29. Steiner RD, Sweetser DA, Rohrbaugh JR, Dowton SB, Toone JR, Applegarth DA. Nonketotic hyperglycinemia: atypical clinical and biochemical manifestations. J Pediatr. 1996:128:243-6.

30. Applegarth DA, Toone JR. Nonketotic hyperglycinemia (glycine encephalopathy): laboratory diagnosis. Mol Genet Metab. 2001;74: 139-46.

31. Schaefer A, Piquard F, Haberey P. Plasma amino-acids analysis: effects of delayed samples preparation and of storage. Clin Chim Acta. 1987;164: 163-9.

32. Sahai $S$, Uhlhaas $S$. Stability of amino acids in human plasma. Clin Chim Acta. 1985;148:255-9.

33. Refsum H, Ueland PM, Svardal AM. Fully automated fluorescence assay for determining total homocysteine in plasma. Clin Chem. 1989;35: 1921-7.

34. Demaster EG, Shirota FN, Redfern B, Goon DJ, Nagasawa HT. Analysis of hepatic reduced glutathione, cysteine and homocysteine by cation exchange high-performance liquid chromatography with electrochemical detection. J Chromatogr. 1984;308:83-91.

35. Magera MJ, Lacey JM, Casetta B, Rinaldo P. Method for the determination of total homocysteine in plasma and urine by stable 
isotope dilution and electrospray tandem mass spectrometry. Clin Chem. 1999;45:1517-22.

36. McCredie KB, Ho DH, Freireich EJ. L-asparaginase for the treatment of cancer. CA Cancer J Clin. 1973;23:220-7.

37. Shrivastava A, Khan AA, Khurshid M, Kalam MA, Jain SK, Singhal PK. Recent developments in L-asparaginase discovery and its potential as anticancer agent. Crit Rev Oncol Hematol. 2016;100:1-10.

38. Knebel B, Strassburger K, Szendroedi J, et al. Specific metabolic profiles and their relationship to insulin resistance in recent-onset type 1 and type 2 diabetes. J Clin Endocrinol Metab. 2016;101:2130-40.

39. Wurtz $P$, Havulinna AS, Soininen $P$, et al. Metabolite profiling and cardiovascular event risk: a prospective study of 3 population-based cohorts. Circulation. 2015;131:774-85.

40. Narayan SB, Ditewig-Meyers G, Graham KS, Scott R, Bennett MJ. Measurement of plasma amino acids by ultraperformance ${ }^{\circledR}$ liquid chromatography. Clin Chem Lab Med. 2011;49:1177-85.

41. Pappa-Louisi A, Nikitas $P$, Agrafiotou P, Papageorgiou A. Optimization of separation and detection of 6-aminoquinolyl derivatives of amino acids by using reversed-phase liquid chromatography with on line UV, fluorescence and electrochemical detection. Anal Chim Acta. 2007;593: 92-97.

42. Vogeser $M$, Seger C. Quality management in clinical application of mass spectrometry measurement systems. Clin Biochem. 2016;49:947-54.

43. Spencer K. Analytical reviews in clinical biochemistry: the estimation of creatinine. Ann Clin Biochem. 1986;23(Pt 1):1-25.

44. Tsikas D, Wolf A, Frolich JC. Simplified HPLC method for urinary and circulating creatinine. Clin Chem. 2004;50:201-3.

45. Takahashi N, Boysen G, Li F, Li Y, Swenberg JA. Tandem mass spectrometry measurements of creatinine in mouse plasma and urine for determining glomerular filtration rate. Kidney Int. 2007;71:266-71.

46. Kaspar H, Dettmer K, Gronwald W, Oefner PJ. Advances in amino acid analysis. Anal Bioanal Chem. 2009;393:445-52.

47. Piraud M, Vianey-Saban C, Petritis K, Elfakir C, Steghens JP, Bouchu D. Ion-pairing reversed-phase liquid chromatography/electrospray ionization mass spectrometric analysis of 76 underivatized amino acids of biological interest: a new tool for the diagnosis of inherited disorders of amino acid metabolism. Rapid Commun Mass Spectrom. 2005;19:1587-602.

48. Kaspar $H$, Dettmer $K$, Chan $Q$, et al. Urinary amino acid analysis: a comparison of iTRAQ-LC-MS/MS, GC-MS, and amino acid analyzer. J Chromatogr B Anal Technol Biomed Life Sci. 2009;877:1838-46.

49. Waterval WA, Scheijen JL, Ortmans-Ploemen MM, Habets-van der Poel CD, Bierau J. Quantitative UPLC-MS/MS analysis of underivatised amino acids in body fluids is a reliable tool for the diagnosis and follow-up of patients with inborn errors of metabolism. Clin Chim Acta. 2009;407:36-42.

50. Le A, Ng A, Kwan T, Cusmano-Ozog K, Cowan TM. A rapid, sensitive method for quantitative analysis of underivatized amino acids by liquid chromatography-tandem mass spectrometry (LC-MS/MS). J Chromatogr B Anal Technol Biomed Life Sci. 2014;944:166-74.

51. Pickering Laboratories. Amino acid analysis part 1. https://www. pickeringlabs.com/library/primers/amino-acid-analysis-part-1/. Accessed 26 December 2016.

52. Davies M. Protein and peptide analysis. Using a dedicated amino acid analyser. The applications book. Cambridge, UK: Biochrom Ltd, 2003.

53. Ross PL, Huang YN, Marchese JN, et al. Multiplexed protein quantitation in Saccharomyces cerevisiae using amine-reactive isobaric tagging reagents. Mol Cell Proteom. 2004;3:1154-69.

54. Dietzen DJ, Weindel AL, Carayannopoulos $\mathrm{MO}$, et al. Rapid comprehensive amino acid analysis by liquid chromatography/tandem mass spectrometry: comparison to cation exchange with post-column ninhydrin detection. Rapid Commun Mass Spectrom. 2008;22:3481-8.

55. Held PK, White L, Pasquali M. Quantitative urine amino acid analysis using liquid chromatography tandem mass spectrometry and aTRAQ reagents. J Chromatogr B Anal Technol Biomed Life Sci. 2011;879: 2695-703.

56. Filee R, Schoos R, Boemer F. Evaluation of physiological amino acids profiling by tandem mass spectrometry. JIMD Rep. 2014;13:119-28.

57. Prinsen HC, Schiebergen-Bronkhorst BG, Roeleveld MW, et al. Rapid quantification of underivatized amino acids in plasma by hydrophilic interaction liquid chromatography (HILIC) coupled with tandem massspectrometry. J Inherit Metab Dis. 2016;39:651-60.

58. William C, Molinaro RJ, Bachmann LM, et al. Clinical and Laboratory Standards Institute (CLSI): Wayne, PA, 2014. https://clsi.org/media/1346/ c62a_sample.pdf.

59. Wang S, Cyronak M, Yang E. Does a stable isotopically labeled internal standard always correct analyte response? A matrix effect study on a LC/MS/MS method for the determination of carvedilol enantiomers in human plasma. J Pharm Biomed Anal. 2007;43:701-7.

60. Piraud $M$, Vianey-Saban C, Petritis $K$, et al. ESI-MS/MS analysis of underivatised amino acids: a new tool for the diagnosis of inherited disorders of amino acid metabolism. Fragmentation study of 79 molecules of biological interest in positive and negative ionisation mode. Rapid Commun Mass Spectrom. 2003:17:1297-311.

61. Burtis CA, Ashwood ER, Bruns DE. Tietz textbook of clinical chemistry and molecular diagnostics. Elsevier Health Sciences: Philadelphia, US, 2012. 